\title{
Evaluation of a momentum based impact model in frontal car collisions for the prospective assessment of ADAS
}

\author{
Stefan Smit ${ }^{1 *}$, Ernst Tomasch¹, Harald Kolk ${ }^{1}$ Michael Alois Plank', Jürgen Gugler ${ }^{1}$ and Hannes Glaser ${ }^{2}$
}

\begin{abstract}
Motivation: The advent of active safety systems calls for the development of appropriate testing methods that are able to assess their capabilities to avoid accidents or lower impact speeds and thus, to mitigate the injury severity. Up to now the assessment is mostly based on the decrease of the collision speed due to CMS (collision mitigation systems). In order to assess the effects on injury severity developing methods, that are able to predict collision parameters correlating with the risk of getting injured, such as delta-v, for different impact situations is a mandatory task.
\end{abstract}

Objective: In this study a momentum based impact model is assessed in terms of reliability to solve the collision mechanics and therefore to predict delta-v for frontal car collisions.

Method: Real accidents were re-simulated using pre-defined input parameters for the impact model (virtual forward simulation - VFS). Subsequently the impact model was analyzed for its sensitivity to specific input parameters.

Conclusion: It was shown that VFS works for full impacts while improvements and optimizations are required for impacts that include a sliding movement in the contact zone of the vehicles.

Keywords: Driver assistance systems, Collision mitigation systems, Effectiveness assessment, Impact mechanics, Point of impact, Momentum based impact model

\section{Introduction}

In 2010 the European Commission [1] released a series of precautionary measures (focusing on vehicle safety, the safety of infrastructure and road users' behavior) to halve the number of road deaths until 2020. Salmon et al. [2] point out that between $75 \%$ and $95 \%$ of all traffic accidents are - at least partially - caused by human error. In this context, the integration of collision mitigation systems (CMS) in new cars is a reasonable step towards enhanced occupant safety. The purpose of CMS is to monitor the surrounding traffic, detect critical events, to brief the driver on the current traffic situation or to take actions if the driver is not responding. Hence, the driver can be partially unburdened from mental stress and his failure

\footnotetext{
* Correspondence: stefan.smit@tugraz.at

${ }^{1}$ Vehicle Safety Institute, Graz University of Technology, Inffeldgasse 23/I,

8010 Graz, Austria

Full list of author information is available at the end of the article
}

probability can be reduced. CMS, such as autonomous emergency braking systems (AEB), aim to mitigate injuries in traffic accidents, usually by reducing the impact velocity and therefore the kinetic energy of the vehicle. However, automatically triggered braking manoeuvers also affect the collision configuration and the impact pattern. E.g. if the overlap in frontal collisions is smaller, a lower delta-v can cause more severe injuries, due to high intrusion to the passenger compartment [3].

A number of different approaches is available to determine the efficacy of CMS - retrospective analysis of real accident data and prospective analysis based either on testing (driving simulator studies, field operational tests, naturalistic driving studies) or simulation (virtual testing). Especially for newer ADAS retrospective analyses are difficult, because the number of accidents involving cars equipped with CMS is still too low to provide results of high statistical significance. Thus, prospective 
investigations are needed. One possibility is to do physical testing, using cars equipped with appropriate systems, similar to the Euro NCAP (New Car Assessment Programme) tests. In these tests, restrictions are applied to reduce the testing effort and to increase the repeatability. E.g. in the Euro NCAP AEB (autonomous emergency braking) testing procedure [4] only the reduction of the collision velocity is considered when calculating the final rating. Instead of physical testing, virtual testing (VT) can be done. VT offers the possibility to simulate a high number of different accident scenarios in a short period of time. Thus, more accident scenarios can be addressed. In VT, it is necessary to introduce surrogate parameters for the injury severity. One of these surrogate parameters is delta-v, the change in velocity between the pre- and post-crash trajectories of a vehicle [5]. It is obtained by resolving the collision mechanics.

In contrast to VT, retrospective analyses use statistical approaches for determining the influence of specific safety systems on the accident and injury occurrence. The basic data for these analyses is usually gathered via accident reconstruction. The main task of accident reconstruction is to reproduce the accident sequence in time as well as in space. Detailed descriptions can be found in Johannsen [6] or Burg and Moser [7]. Accident reconstruction utilizes data such as the damage patterns, the final positions of the vehicles, etc. Usually it is necessary to tune the pre-impact collision parameters (e.g. point of impact (POI), coefficient of restitution, etc.) after setting up the simulation to obtain the final positions of the vehicles. The final vehicle positions and/or an estimation of EES (energy equivalent speed [8]) can thus be used to check the plausibility of the results [9]. The reconstruction provides data like impact velocities, post- crash movements or delta-v.
VT can be done using the so called virtual forward simulation (VFS). VFS can be used to simulate traffic accidents, starting within the pre-crash phase (with a sufficient time history before the collision, so that the CMS can take actions [10]). Within VFS, the vehicles can be equipped with generic ADAS to simulate their behavior. Studies carried out by Zauner et al. [11], Tomasch et al. [10] or Kolk et al. [12,13] used the method of VFS to rate the effects of CMS. Hence the collision configuration, like the relative vehicle positions or the impact velocities, are manipulated by the CMS it is necessary to predict the pre-impact collision parameters for the impact model. The collision mechanics are then solved using these pre-impact parameters. In VFS, the data of a real accident i.e. final vehicle positions, damage patterns, damage depth, etc. are not known. Thus, they cannot be used to validate the results of the simulation.

To understand the problem of VFS, an example of a real accident is used to explain the possible differences between reconstruction and VFS. Due to lack of information, the input-parameters of the impact model are set to the default values in case of VFS. The first difference refers to the collision configuration, shown in Fig. 1(a). Because the penetration time (time from the initial penetration until the exchange of momentum) must be estimated in VFS, the distance between the COGs (center of gravity) of the vehicles increases by $0.23 \mathrm{~m}$ in VFS. Thus, the relative POI (point of impact) positions within the vehicles also change $(0.26 \mathrm{~m}$ for vehicle 1 and $0.02 \mathrm{~m}$ for vehicle 2). The post-crash movements of the two vehicles in the reconstruction and in the VFS are completely different, see Fig. 1(b). The differences between the final vehicle positions are $41 \mathrm{~m}$ in $\mathrm{x}$ - and $7.3 \mathrm{~m}$ in $\mathrm{y}$-direction for V1 and $2.4 \mathrm{~m}$ (x-direction) and $9.8 \mathrm{~m}$ (y-direction) for $\mathrm{V} 2$. (a)

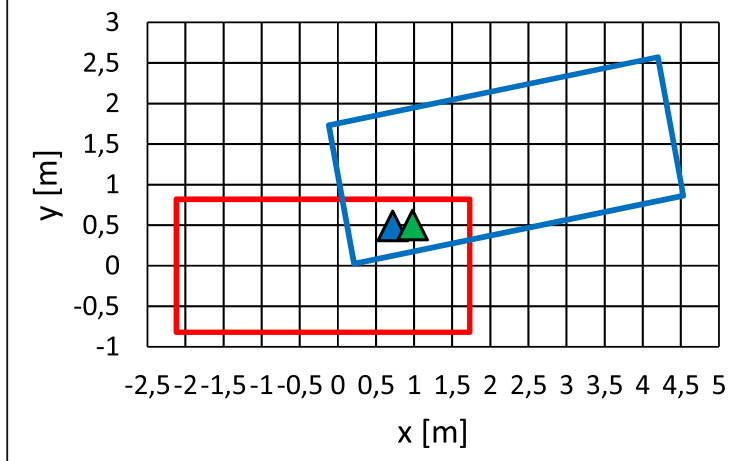

$\longrightarrow \mathrm{V} 1-\mathrm{V} 2 \Delta \mathrm{POI}$ reconstruction $\Delta$ POIVFS (b)

Post-crash movement

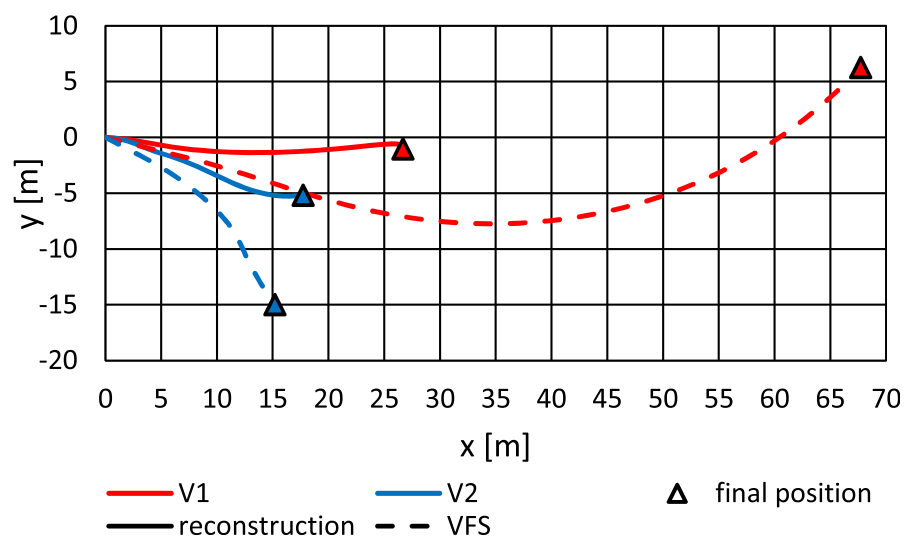

Fig. 1 Differences between reconstruction and VFS: (a) Collision configuration, (b) Post-crash movement of the vehicles 


\section{Objective}

The first objective of this study is to assess the performance of a momentum based impact model in terms of accuracy of the post-crash parameters e.g. delta-v, post-crash velocity or final positions of the vehicles. The second objective is to analyze the model on its sensitivity to its input parameters, like initial speeds, position of the POI, angle of the contact plane (CP), coefficient of restitution or coefficient of friction.

\section{Method}

\subsection{Momentum based impact model}

The general purpose of impact modelling is to determine the post-impact conditions of the system, proceeding from given initial conditions (pre-impact conditions). The impact model used in this study is based on the conservation of linear and angular momentum during the crash phase. Detailed descriptions can be found in Steffan and Moser [14], Gilardi and Sharf [15] Brach and Brach [16], Burg and Moser [7] or Appel et al. [17]. The basic formulations of this model are based on Newton's $1^{\text {st }}$ and $2^{\text {nd }}$ law and were first used by Kudlich [18], Slibar [19], to analyze vehicle collisions. The model is capable of computing the post-crash parameters (velocities, yaw rates, etc.) directly from the pre-impact conditions. In order to keep the computational effort low, the following assumptions are made:

- The crash force between the interacting vehicles is exchanged in one discrete point - the POI

- Tire forces and the gravitational force are neglected during the crash

- The duration of the crash phase is infinitely small, therefore no acceleration pulses or vehicle deformations are computed

- The vehicles do not move during the crash phase

- The vehicle bodies have linear stiffness behavior

\subsubsection{Conservation of momentum - Equations}

The conservation of momentum can be derived by combining Newton's $2^{\text {nd }}$ and $3^{\text {rd }}$ law and states that the total momentum in a closed system of $n$ interacting bodies remains constant, if no external forces are applied. The total momentum equals the sum of momentums $\overrightarrow{p_{i}}$ of the individual bodies (1):

$$
\vec{p}=m \vec{v}=\overrightarrow{p_{1}}+\overrightarrow{p_{2}}+\ldots+\overrightarrow{p_{n}}=\sum_{i=1}^{n} \overrightarrow{p_{i}}=\text { const }
$$

The same equation must also be satisfied by the angular momentum $\vec{L}(2)$ :

$$
\vec{L}=\overrightarrow{L_{1}}+\overrightarrow{L_{2}}+\ldots+\overrightarrow{L_{n}}=\sum_{i=1}^{n} \overrightarrow{L_{i}}=\text { const }
$$

Analyzing equations (1) and (2) shows that the total momentum after the collision is still the same, but the energy is transformed and transferred. During the first phase of the collision, the kinetic energy is partially transformed into vehicle deformation (phase of compression). As in most physical systems, some of the deformation is retransferred into kinetic energy. This is called restitution. Thus, the collision can be split in two phases - the phase of compression and the phase of restitution. The compression phase extends from the initial contact of the vehicles until the velocities of the contact points in normal direction are equal. The latter extends from the end of compression until the bodies separate again. Following this definition, the momentum for each phase can be obtained by:

$$
S_{C}=\int_{t_{0}}^{t_{1}} \vec{F}(t) d t
$$

Equation (3) shows the calculation of the total momentum during the compression phase, where $\vec{F}(t)$ represents the acting force and $t_{0}$ and $t_{1}$ represent the beginning and the end of the compression phase. The momentum for the restitution phase $S_{R}$ can be evaluated using $t_{1}$ and $t_{2}$. At time $t_{2}$ the vehicles separate again. To incorporate the elastic deformation the impulse ratio $\varepsilon$ (coefficient of restitution) is introduced as the ratio between $S_{R}$ and $S_{C}(4)$. Using this definition, the entire exchanged momentum can be calculated as shown in (5).

$$
\varepsilon=\frac{S_{R}}{S_{C}}
$$

$$
\mathrm{S}=S_{C}+S_{R}=S_{C}(1+\varepsilon)
$$

Taking the conservation of energy into account, there are two limits for the impulse ratio. The first one is $\varepsilon=0$ representing a totally plastic impact, the second one is $\varepsilon$ $=1$ referring to a totally elastic impact. In car accidents usually a default value of $\varepsilon=0.1$ is used, because it is applicable for a wide range of closing speeds. This value depends on the geometry of the bodies, the closing speed and the material properties $[6,15,20]$. Additionally the POI, the angle of the CP and the coefficient of friction in the contact zone must be defined.

The penetration depth can be obtained from the damage patterns of the vehicles. To calculate the post-crash parameters (velocities, yaw angles, etc.) a local coordinate system 


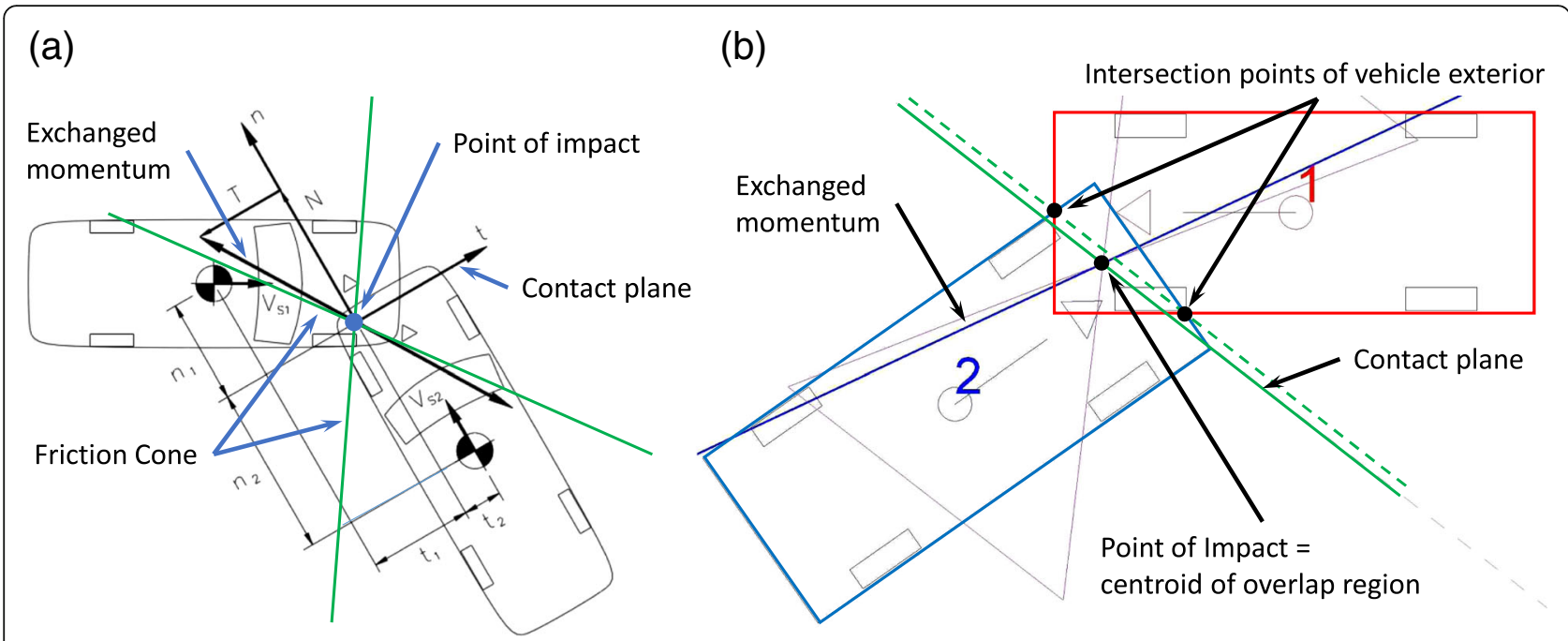

Fig. 2 Momentum based impact model (a) [37] and automatic definition of the POI and the CP (b) [13]

is erected in the POI (see: Fig. 2) and the acting forces are determined. Also a CP is defined, which specifies the direction of the movement if sliding occurs. The definition of the $\mathrm{CP}$ influences the magnitude of the force components $N$ (normal force) and $T$ (friction force) and the corresponding levers $n_{i}$ and $t_{i}$, responsible for the acting torque. The direction of the $\mathrm{CP}$ is crucial for the type of impact. Non-sliding (full) impacts or sliding impacts can occur.

In accident reconstruction the pre-impact parameters of the impact model (collision velocities, POI, angle of the $\mathrm{CP}$, coefficient of restitution, coefficient of friction) need to be refined in a manner, that the vehicles reach their final positions. Additionally estimated parameters such as the EES value can be used to verify the results.

\subsubsection{Full impact}

A full impact occurs when the condition $T \leq \mu N$ is satisfied, meaning that the collision partners do not slide along each other in the contact zone. It also means that the contact points reach the same velocity at the end of the compression phase in normal and tangential direction. In this case, the angle of the $\mathrm{CP}$ does not influence the final results.

\subsubsection{Sliding impact}

The second option is that - in theory $-T>\mu N$ holds. Thus this is not possible, $T=\mu N$ holds, meaning that a sliding movement in the contact zone of the vehicles occurs. As a result, the contact points do not reach a common velocity in tangential direction at the end of the compression phase. In this case, the definition of the $\mathrm{CP}$ and the coefficient of friction must be estimated very carefully, because the results (final positions, trajectories) show a high dependency on these parameters [21]. Sliding impacts are identified as follows. The opening angle of the friction cone is calculated according to equation (6).

$$
\rho=\arctan (\mu)
$$

If the angle between crash force and normal direction $n$ in Fig. 2(a) is equal to the opening angle of the friction cone $\rho$, a sliding impact occurs.

Many studies have been carried out to evaluate the suitability of this impact model for accident reconstruction. Bailey et al. [22] staged five different collisions and recorded data such as delta-v, post-crash trajectories, impact speeds etc. After the tests, they validated the impact models using two different methods. First, they calculated the input parameters for the model from the recorded test data and compared the final positions and post-crash trajectories. Second, they used the final positions and post-crash trajectories to determine the initial conditions. Finally, they compared the obtained results with the measured data and found that the error of the model stays within reasonable limits. Other investigations on this topic were carried out by Cliff and Montgomery [23] and Cliff and Moser [24].

\subsection{Virtual forward simulation}

The VFS method has become more popular for the assessment of ADAS in recent years. One of the advantages of VFS is that a large number of scenarios can be evaluated in a short period of time. When implementing generic ADAS into a vehicle in VFS, the simulation starts within the pre-crash phase to be able to simulate the behavior of the ADAS during the pre-crash phase. In 
case the ADAS takes action, the collision configuration (velocities, impact pattern, etc.) might change. The location of the POI and the angle of the $\mathrm{CP}$ might thus also be changed. Additionally it is not possible to use final positions or estimation of EES to verify the results (as these parameters could be used in accident reconstruction). Therefore, the user has to find a way of determining reasonable values for the input parameters (POI, CP, coefficient of restitution, etc.) of the impact model.

In this study, the VFS was done using the simulation tool X-RATE (Extended Effectiveness Rating of Advanced Driver Assistance Systems) developed by Kolk [25]. X-RATE is a control platform that is capable of setting up driving dynamics simulations and run them automatically. It is based on MATLAB ${ }^{\circ}$ and utilizes ${\mathrm{PC}-\mathrm{Crash}^{\mathrm{Tx}}}^{\mathrm{as}}$ as solver for the vehicle dynamics and collision mechanics. The crash related parameters are then gathered and saved.

\subsubsection{Definition of the $\mathrm{POI}$ and the $\mathrm{CP}$ using geometrical rules}

For reasons of simplicity, the vehicle outline is represented by a rectangle with the same length and width as the real vehicle. As required by the impact model, the vehicles penetrate each other before they reach the collision configuration and the momentum is exchanged. The overlap region, shown in Fig. 2 (b), is represented by a polygon that can be calculated using the algorithm of Sutherland and Hodgman [26]. The corners of this polygon either represent the corners of the vehicle or the intersection points between the vehicle outlines. The centroid of the overlap region can be obtained as described by Nürnberg [27]. The area of the overlap polygon and the coordinates of the nodes of the polygon are used to obtain the coordinates of the centroid. In addition to the POI the $\mathrm{CP}$ is also defined using geometrical rules only. The direction of the $\mathrm{CP}$ is defined by the intersection points of the simplified vehicle contours. It is then translated in parallel so that it is coincident with the POI, see Fig. 2 (b).

\section{Material}

The in-depth accident database CEDATU (Central Database for In-Depth Accident Study) developed by Tomasch $[28,29]$ was the source for the basic accident data. The CEDATU data fields are based on the STAIRS (Standardization of Accident and Injury Registration Systems) protocol [30]. The fields were enhanced and extended in accordance with the results obtained in different research projects, such as PENDANT (Pan-European Coordinated Accident and Injury Databases, [31]), RISER (Roadside Infrastructure for Safer European Roads, [32]) and ROLLOVER (Improvement of Rollover Safety for Passenger Vehicles, [33]). Additionally data fields of the national statistics in Austria are incorporated to make a direct comparison possible [34].

\section{Sampling criteria}

The following sampling criteria were applied for this study:

- Only fatalities involving two passenger cars proceeding in opposite directions

- No subsequent collisions of the vehicles when moving to the final positions

- The penetration depth is set constant

- Frontal damage of the vehicles (according to the STAIRS protocol [30])

- PDoF (Principal Direction of Force): 1, 2, 10, 11, 12 (STAIRS protocol [30])

- Random selection of accidents - no frequency correlation with national statistics (bias of results)

Figure 3 shows the six different accident scenarios considered and their frequency after applying the sampling criteria. The classification is based on the Austrian National Traffic Accident Statistics [35]. Frontal collisions on straight roads (scenario 241) were the most frequent accident scenarios, followed by frontal collisions in bends (scenario 242) and frontal collisions with oncoming traffic while overtaking (scenario 264).

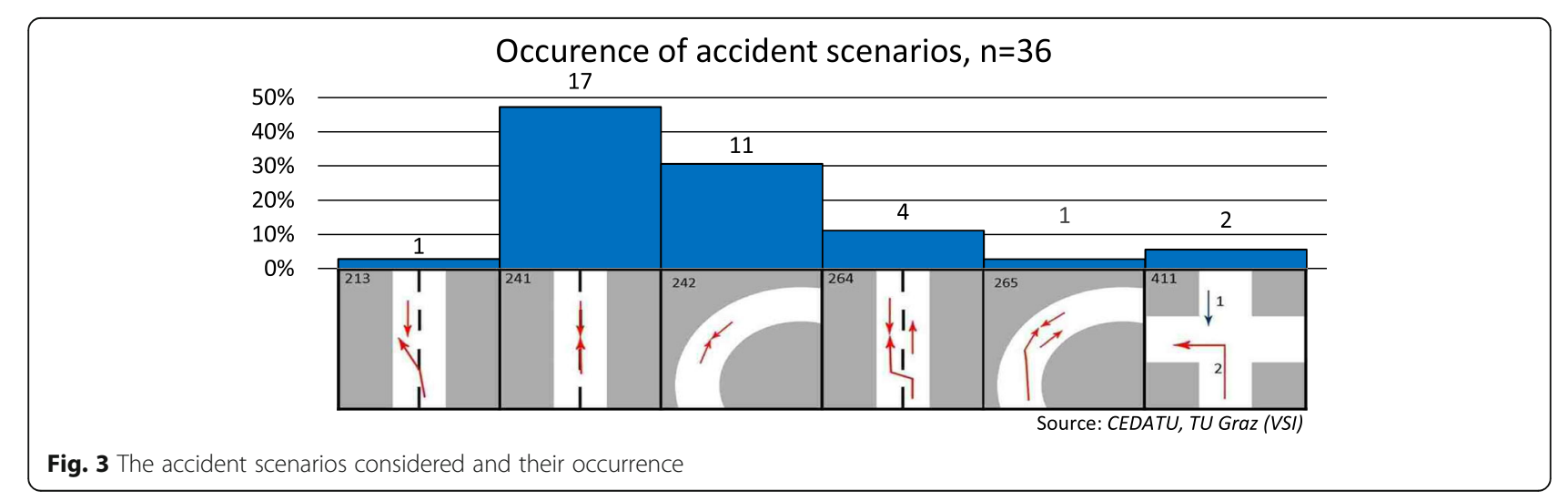




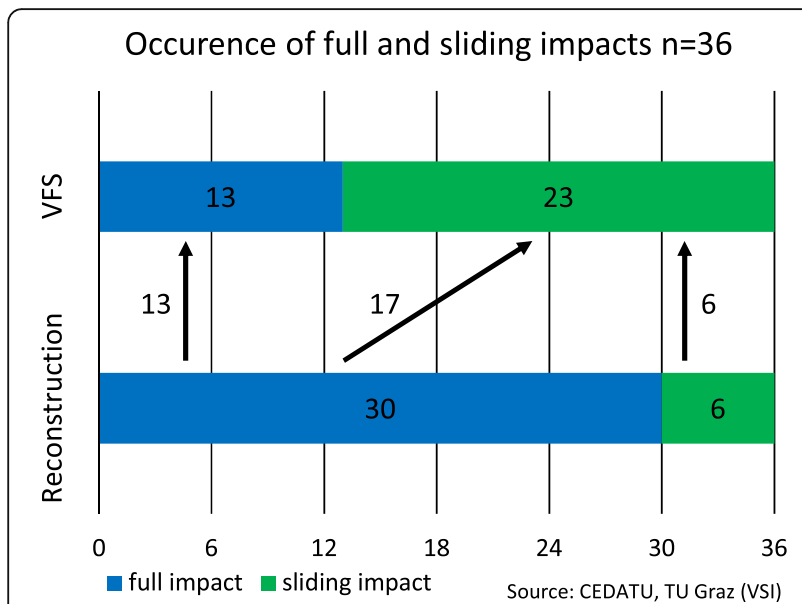

Fig. 4 Occurrence of full and sliding impacts

All of the accidents were reconstructed and re-simulated by VFS. Thirty-six accidents involving 72 vehicles were analyzed.

\section{Results and discussion}

Figure 4 shows the occurrence of full and sliding impacts in reconstruction and VFS as well as the changes (arrows) from full to sliding impacts. It can be seen that the VFS (POI and CP defined by geometrical rules) leads to more sliding impacts than the accident reconstruction. Using a Chi-squared test [36] shows that the difference is highly significant $(p \leq 0.01)$. Because the accident reconstruction can make use of the actual damage patterns and final positions of the involved vehicles to optimize the POI and the CP, the detection of the impact type is easier than in VFS. Usually a sliding impact is expected to be more likely if the overlap (width of the damaged area divided by the vehicle width) is smaller. Analyzing the reconstructed accidents, this can be validated, because the mean overlap is $65.5 \%$ for full impacts compared to $32.5 \%$ for sliding impacts. Using VFS the results are different. Here the mean overlap for full impacts is $77 \%$ and the mean overlap of sliding impacts is $50.3 \%$. A number of 13 accidents led to full impacts in reconstruction as well as in VFS. Six accidents were identified as sliding impacts for both simulations. Calculating the difference results in a number of 17 collisions, which show different results for reconstruction and VFS.

The relative Position of the POI within the vehicle is shown in Fig. 5. When applying the method of accident reconstruction, the POIs for full impacts (blue circles) are distributed across the whole width, while the ones for the sliding impacts (green circles) are close to the left side of the vehicle. The cause of this is the regulation for driving on the right side of the road. Therefore, impacts with small overlaps mostly affect the left side of the vehicle. For VFS the POIs for both - full and sliding impacts (red and yellow triangles) - are randomly distributed across the whole width of the vehicle. Additionally the distances between the POIs were analyzed. The mean deviation over all vehicles is $0.190 \mathrm{~m}$ $(n=72)$. When both simulations resulted in full impacts the mean distance between the POIs is $0.167 \mathrm{~m}(n=$ $26)$, compared to $0.148(n=12)$ for sliding impacts. The distance between the POIs was the largest when the type of impact (full or sliding impact) changed between the simulation runs $(0.222 \mathrm{~m}, n=34)$.

In the context of the final positions in full impacts, similar results are obtained for reconstruction and VFS. For sliding impacts there is quite a big difference. Using VFS, the post-crash movement is shorter than in reconstruction.

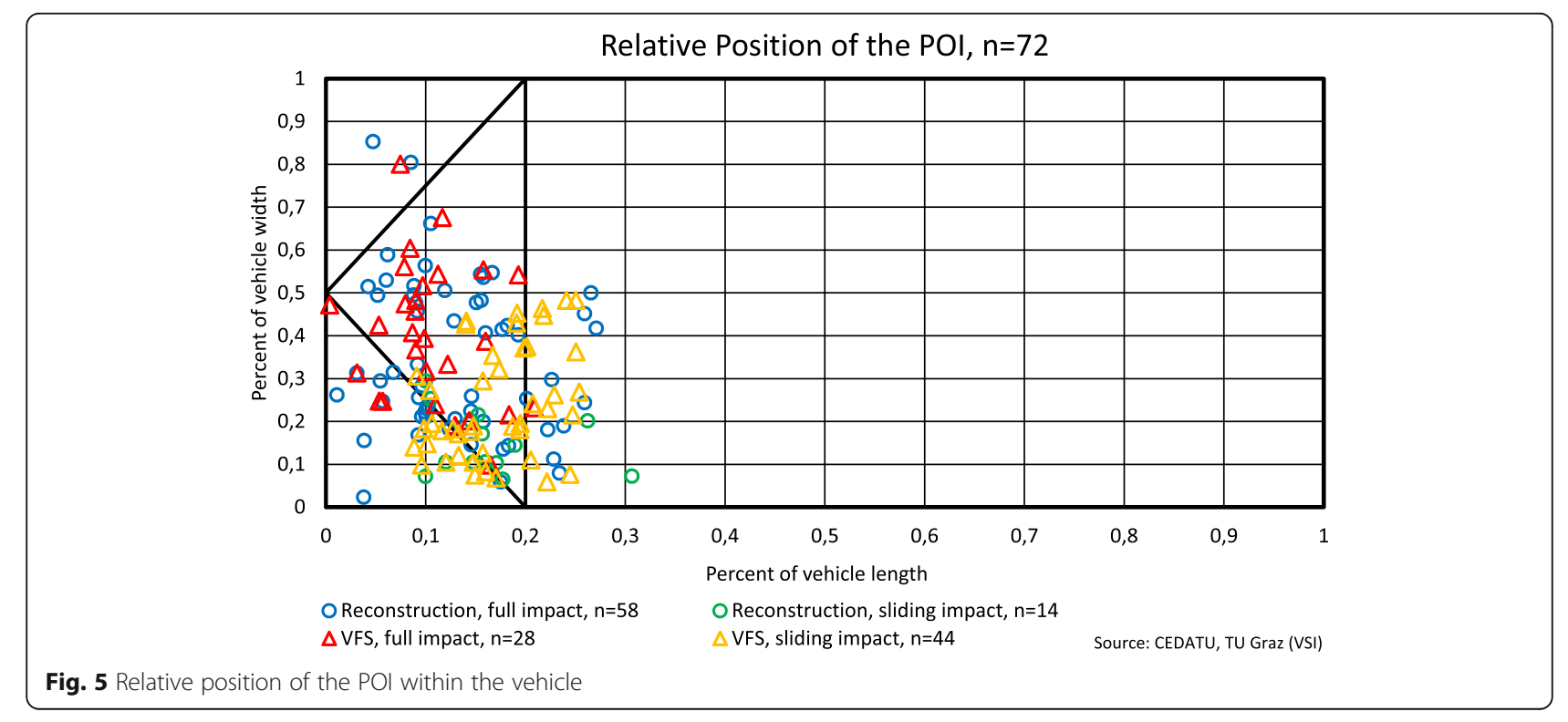




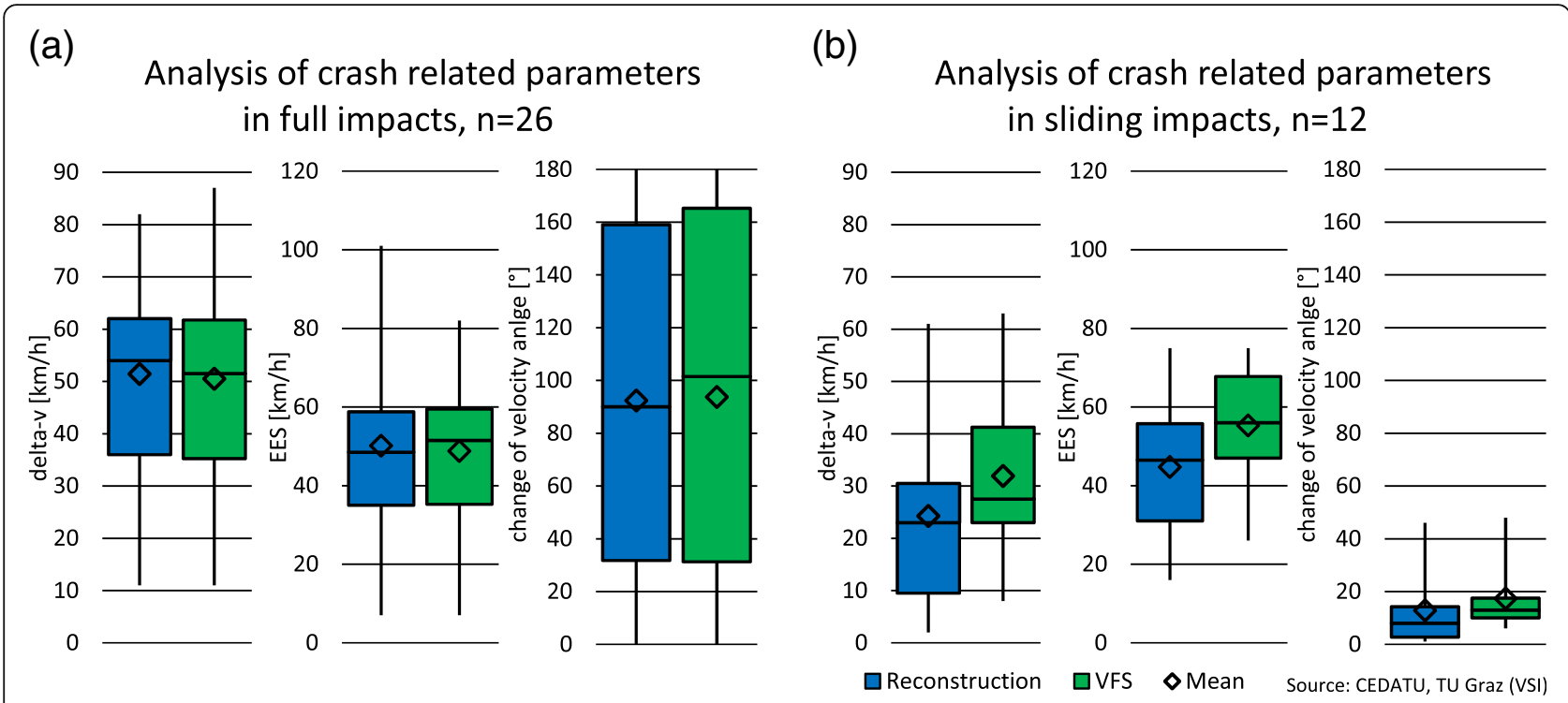

Fig. 6 Differences of crash related parameters (delta-v, EES, change of velocity angle) in full (a) and sliding impacts (b)

Figure 6 shows boxplots for different crash related parameters for full (a) and sliding (b) impacts. The whiskers show the minimal and maximal values of the corresponding parameter. Due to the fact that equal axes scaling is used for Fig. 6 (a) and (b) the different behavior of full and sliding impacts can be seen. Therefore, the validity of the VFS using geometrical rules to predict the POI and the CP is analyzed separately.

Table 1 shows the results of paired t-tests [36] for the parameters shown in Fig. 6. To enable a better comparison of the results the samples were limited to collisions that led to the same impact type (full or sliding) in both simulations (reconstruction and VFS). Given the results in Table 1, it can be said that the results of the VFS are similar to the ones of the reconstruction when considering full impacts. As the $p$-values are far above the threshold of 0.05 , the hypothesis that there are significant differences of the mean values can be neglected for all three parameters (delta-v, EES, change of velocity angle). Thus, the definition of the POI and $\mathrm{CP}$ by using geometrical rules can be applied for full impacts.
When looking at sliding impacts, the hypothesis that the mean values are different (for any of the three parameters) cannot be neglected $(p>0.05)$, although the differences appear to be large when examining the plots of Fig. 6b. However, as the number of samples is relatively low, the validity of the test is also low. When comparing the results, however, it can be stated that the differences are much larger than in full impacts. Thus, a determination of the POI and the $\mathrm{CP}$ in sliding impacts by geometrical rules only, could be non-valid.

\section{Conclusion}

The results of this study show that the use of VFS is reasonable for full impacts. The comparison between accident reconstruction and VFS showed that the differences concerning the final positions of the vehicles, delta-v, EES and the change of the velocity angle are small. Due to the good results for the delta-v value, an integration of risk functions based on delta-v is possible. This study also shows a good correlation with the results of Kolk et al. [14], where the same impact model was investigated for junction accidents.

Table 1 Results for the paired-t-tests applied for the values of Fig. 6

\begin{tabular}{|c|c|c|c|c|}
\hline & Number of samples $n$ & Mean value reconstruction & Mean value VFS & $p$-value \\
\hline Delta-v, full impacts [km/h] & 26 & 51.42 & 50.54 & 0.482 \\
\hline EES, full impacts $[\mathrm{km} / \mathrm{h}]$ & 26 & 50.19 & 48.85 & 0.398 \\
\hline Change of velocity angle, full impacts $\left[^{\circ}\right]$ & 26 & 92.42 & 93.71 & 0.723 \\
\hline Delta-v, sliding impacts [km/h] & 12 & 24.25 & 31.92 & 0.255 \\
\hline EES, sliding impacts [km/h] & 12 & 44.75 & 55.33 & 0.180 \\
\hline Change of velocity angle, sliding impacts $\left[^{\circ}\right]$ & 12 & 12.83 & 17.33 & 0.301 \\
\hline
\end{tabular}


For sliding impacts, the use of VFS requires optimization and improvements. Within the comparison of accident reconstruction and VFS the final vehicle positions and delta-v showed a high sensitivity to the definition of the POI and the $\mathrm{CP}$ and thus were quite different. This is due to the fact, that the angle of the contact plane has a great influence on the normal and tangential forces acting in the POI as well as on the levers for the angular momentum.

\section{Outlook}

Future research should also include the investigations on the penetration depth that was set to a fixed value of 30 $m s$ in this study. The penetration influences the levers of the exchanged angular momentum in the impact model. In reconstruction, the depth of penetration can be obtained from the damage patterns. In VFS, it would be necessary to find a correlation between the closing speed, the overlap and the penetration depth to be used.

Further, the algorithm for the determination of the POI and the CP in VFS needs further investigations specifically because the use of geometrical relationships often results in sliding impacts being identified instead of full impacts.

The prediction of the POI and $\mathrm{CP}$ for sliding impacts must be analyzed in detail, because these impacts show a very sensitive behavior. In addition, the number of accidents investigated should be increased to provide results that are more reliable. Additionally, the analyses carried out in this context should also be applied for other types of vehicles and accident scenarios.

An ANOVA could be used to find the most sensitive pre-collision impact parameters in order to incorporate multiple parameters i.e. overlap, POI, angle of the CP, penetration depth, masses of the vehicles and others in the sensitivity analysis.

\section{Abbreviations \\ ADAS: Advanced driver assistance system; AEB: Autonomous emergency braking; CEDATU: Central Database for In-Depth Accident Study; CMS: Collision mitigation system; COG: Center of gravity; CP: Contact plane; CS: Coordinate system; EES: Energy equivalent speed; NCAP: New Car Assessment Programme; POI: Point of impact; VFS: Virtual forward simulation; VT: Virtual testing; X-RATE: Extended Effectiveness Rating of Advanced Driver Assistance Systems}

\section{Acknowledgements}

Not applicable.

\section{Funding}

Not applicable.

Availability of data and materials

Not applicable.

Authors' contributions

All authors read and approved the final manuscript.

Competing interests

The authors declare that they have no competing interests.

\section{Publisher's Note}

Springer Nature remains neutral with regard to jurisdictional claims in published maps and institutional affiliations.

\section{Author details}

${ }^{1}$ Vehicle Safety Institute, Graz University of Technology, Inffeldgasse 23/I, 8010 Graz, Austria. ${ }^{2}$ Consultant for accident reconstruction, Limbergstraße 54, 3500 Krems a.d. Donau, Austria.

Received: 13 June 2018 Accepted: 19 December 2018

Published online: 04 January 2019

\section{References}

1. Minsart, N. (2010). Road Safety Programme 2011-2020: detailed measures.

2. Salmon, P., Regan, M., \& Johnston, I. (2005). Human error and road transport: Phase one - A framework for an error tolerant road transport system, Victoria.

3. Rudd, R. W., Scarboro, M., \& Saunders, J. (2011). Injury Analysis of Real-World small overlap and oblique Frontal Crashes. In 22nd International Technical Conference on The Enhanced Safety of Vehicles (ESV), Paper No 11-0384, Washington.

4. Euro, N. C. A. P. (2015). European New Car Assessment Programme: Test protocol - AEB systems. Version 1.1.

5. Day, T. D., \& Hargens, R. L. (1987). An Overview of the Way EDCRASH Computes Delta-V. In SAE International Congress and Exposition. Warrendale: SAE International400 Commonwealth Drive.

6. Johannsen, H. (2013). Unfallmechanik und Unfallrekonstruktion: Grundlagen der Unfallaufklärung, 3., überarbeitete Auflage. ATZ/MTZ-Fachbuch. Wiesbaden: Springer Vieweg.

7. Burg, H., \& Moser, A. (2017). Handbuch Verkehrsunfallrekonstruktion: Unfallaufnahme, Fahrdynamik, Simulation, 3. Aufl. 2017. ATZ/MTZ-Fachbuch.

8. Burg, H., \& Zeidler, F. (1980). EES -Ein Hilfsmittel zur Unfallrekonstruktion und dessen Auswirkungen auf die Unfallforschung. In Der Verkehrsunfall (Vol. 18, p. 75).

9. Wach, W., \& Unarski, J. (2007). Uncertainty of calculation results in vehicle collision analysis. Forensic Sci Int, 167(2-3), 181-188.

10. Tomasch, E., Kolk, H., Sinz, W., et al. (2015). Prospektive Bewertung der Kollisionsschwere von L6e Fahrzeugen unter Berücksichtigung eines Kollisionsminderungssystems. In 10. VDI-Tagung Fahrzeugsicherheit Sicherheit 2.0: Berlin, 25. und 26. November 2015, Nichtred. Ms.-Dr, vol 2265 (pp. 407-418). Düsseldorf: VDIVerlag.

11. Zauner, C., Tomasch, E., Sinz, W., et al. (2014). Assessment of the effectiveness of Intersection Assistance Systems at urban and rural accident sites. In ESAR - Expert Symposium on Accident Research.

12. Kolk, H., Kirschbichler, S. K., Tomasch, E., et al. (2016). Prospective evaluation of the collision severity of L7e vehicles considering a Collision Mitigation System. In: Transportation Research Procedia. Elsevier.

13. Kolk, H., Sinz, W., Tomasch, E., et al. (2016). Evaluation of a momentum based impact model and application in an effectivity study considering junction accidents. In ESAR (ed) 7th International Conference on ESAR "Expert Symposium on Accident Research".

14. Steffan, H., \& Moser, A. (1996). The Collision and Trajectory Models of PCCRASH. In International Congress \& Exposition. SAE International.

15. Gilardi, G., \& Sharf, I. (2002). Literature survey of contact dynamics modelling Mech Mach Theory, 37(10), 1213-1239. https://doi.org/10.1016/S0094$114 \times(02) 00045-9$.

16. Brach, R. M., \& Brach, R. M. (2011). Vehicle accident analysis and reconstruction methods (2nd ed.). Warrendale: SAE International.

17. Appel, H., Krabbel, G., \& Vetter, D. (2002). Unfallforschung, Unfallmechanik und Unfallrekonstruktion, 2.Auflage. Wiesbaden: Vieweg+Teubner Verlag.

18. Kudlich, H. (1966). Beitrag zur Mechanik des Kraftfahrzeug-Verkehrsunfalls. Wien: Techn. Hochsch., Dissertation.

19. Slibar, A. (1966). Die mechanischen Grundsätze des Stoßvorganges freier und geführter Körper und ihre Anwendung auf den Stoßvorgang von Fahrzeugen. In Archiv für Unfallforschung, 2. Jg., H (Vol. 1, p. 31).

20. Antonetti, V. W. (1998). Estimating the Coefficient of Restitution of Vehicle-toVehicle Bumper Impacts, Warrendale, PA

21. Steffan, H., \& Moser, A. (1998). Automatic Optimization of Pre-Impact Parameters Using Post-Impact Trajectories and Rest Positions. SAE technical papers, 980373, 22.

22. Bailey MN, Lawrence JM, Fowler SJ et al. (2000) Data from Five Staged Car to Car Collisions and Comparison with Simulations, Warrendale, PA. 
23. Cliff, W. E., \& Montgomery, D. T. (1996). Validation of PC-Crash - A Momentum-Based Accident Reconstruction Program, SAE Technical Paper 960885, https://doi.org/10.4271/96088.

24. Cliff, W. E., \& Moser, A. (2001). Reconstruction of twenty staged collisions with PC-Crash's optimizer.

25. Kolk, H. (2016). X-RATE: Extended Effectiveness Rating of Advanced Driver Assistance Systems. University of Technology Graz: Vehicle Safety Institute.

26. Sutherland, I. E., \& Hodgman, G. W. (1974). Reentrant Polygon Clipping, New York.

27. Nürnberg, R. (2013). Calculating the area and centroid of a polygon in 2D

28. Tomasch, E. (2007). Entwicklung und systematische Verwertung einer In-depth Datenbank tödlicher Verkehrsunfälle.

29. Tomasch, E., Steffan, H., \& Darok, M. (2008). Retrospective accident investigation using information from court. In TRA (ed) Transport Research Arena Europe 2008 (TRA)

30. Ross, R., Thomas, P., Sexton, B., et al. (1998). An Approach to the Standardisation of Accident and Injury Registration Systems (STAIRS) in Europe. In 16th International Technical Conference on the Enhanced Safety of Vehicles (pp. 1298-1305)

31. Morris, A., \& Thomas, P. (2003). PENDANT - Pan-European Co-ordinated Accident and Injury Databases. In NHTSA (ed) The 18th ESV Conference Proceedings.

32. RISER (2006) Roadside Infrastructure for Safer European Roads. Final Report. EC FP5 project RISER.

33. Gugler, J., \& Steffan, H. (2005). ROLLOVER - Improvement of Rollover Safety for Passenger Vehicels: Final report.

34. Statistik Austria (2007) Erläuterungen und Definitionen zum Zählblatt über einen Straßenverkehrsunfall.

35. Statistik Austria (2000) Unfalltypenkatalog, Wien.

36. Mittag, H.-J. (2012). Statistik: Eine interaktive Einführung, 2. Aufl. 2012. SpringerLehrbuch. Berlin, Heidelberg: Springer.

37. Moser, A. PC-Crash. (2013). Accident-Simulation Program, Operating Manual, Version 10.0 Dr. Steffan Datentechnik Ges.m.b.H., Linz, Austria.

\section{Submit your manuscript to a SpringerOpen ${ }^{\circ}$ journal and benefit from:}

- Convenient online submission

- Rigorous peer review

- Open access: articles freely available online

High visibility within the field

- Retaining the copyright to your article

Submit your next manuscript at $\boldsymbol{\sim}$ springeropen.com 